\title{
LOWER BOUNDS FOR SOLUTIONS OF HYPERBOLIC INEQUALITIES IN UNBOUNDED REGIONS ${ }^{1}$
}

\author{
AMY C. MURRAY
}

\begin{abstract}
This paper considers $C^{2}$ solutions $u=u(t, x)$ of the differential inequality $|L u| \leqq k_{1}(t, x)|u|+k_{2}(t, x)\|\nabla u\|$. The coefficients of the hyperbolic operator $L$ depend on both $t$ and $x$. Explicit lower bounds are given for the energy of $u$ in a region of $\boldsymbol{x}$-space expanding at least as fast as wave-fronts for $L$. These bounds depend on the asymptotic behavior of $k_{1}, k_{2}$, and the coefficients of $L$. They do not require boundary conditions on $u$.
\end{abstract}

1. Introduction. Let $L$ be a hyperbolic operator of the form $L u=$ $A(t, \boldsymbol{x}) u-u_{t t}$ where $A(t, \boldsymbol{x})$ denotes a second order uniformly elliptic operator whose coefficients depend on the time variable $t$ as well as the spatial coordinates $x=\left(x_{1}, \cdots, x_{N}\right)$. Several authors [1], [3], [4], [5], [6] have considered the asymptotic behavior of solutions of the equation

$$
L u=F(t, \boldsymbol{x}, u, \nabla u) .
$$

Because of both the time-dependence in $A(t, x)$ and the presence of $u_{t}$ on the right side, one cannot expect all solutions of (1.1) to behave like solutions of the wave equation.

In [4], the author discussed the asymptotic behavior of $C^{2}$ solutions of the inequality

$$
|L u| \leqq k_{1}(t, \boldsymbol{x})|u|+k_{2}(t, \boldsymbol{x})|\nabla u| .
$$

Such an inequality arises from (1.1) if $F$ is assumed Lipschitz in its last two arguments. The results of [4] establish a kind of unique continuation at infinity, e.g., if a solution of (1.2) decays fast enough inside a forward characteristic conoid for $L$, then it must vanish there. This paper sharpens [4] by providing explicit lower bounds for nonvanishing solutions.

The bounds are comparable to those found by Ogawa [5] for the inequality $\|L u\| \leqq k(t)\|\nabla u\|$ where $\|\cdot\|$ denotes the $L^{2}$ norm on a domain

Received by the editors July 26, 1971 and, in revised form, April 5, 1972.

AMS (MOS) subject classifications (1970). Primary 35B40, 35L99; Secondary 35B45.

Key words and phrases. Hyperbolic inequalities, maximal rates of decay, a priori inequalities.

${ }^{1}$ Research supported in part by Air Force Grant AF-AFOSR 68-1462 and in part by NSF Grant GP 28443.

(c) American Mathematical Society 1973 
in $\boldsymbol{R}^{N}$. Ogawa's bounds improved certain maximal rate of decay results of Protter [6].

Recently Bloom and Kazarinoff [1] have announced upper bounds on solutions of $L u=0$ in expanding regions outside an obstacle.

The operator $A(t, x)$ we consider is defined by

$$
A(t, \boldsymbol{x}) u=\sum_{i j=1}^{N} \frac{\partial}{\partial x_{i}} a_{i j}(t, \boldsymbol{x}) \frac{\partial u}{\partial x_{j}} .
$$

We assume that the coefficients $a_{i j}$ are $C^{\mathbf{1}}$ functions on the half-space $\mathscr{H}=$ $\boldsymbol{R}^{\perp} \times \boldsymbol{R}^{N}$ with $a_{i j}=a_{j i}$. Further, we assume that there are positive constants $m$ and $M$ such that

$$
m^{2} \leqq \sum_{i j=1}^{N} a_{i j}(t, \boldsymbol{x}) \xi_{i} \xi_{j} \leqq M^{2}
$$

for all $(t, \boldsymbol{x}) \in \mathscr{H}$ and all unit vectors $\xi$ in $\boldsymbol{R}^{N}$. Thus the bilinear form

$$
((b, c))=b_{0} c_{0}-\sum_{i j=1}^{N} a_{i j}(t, x) b_{i} c_{j}
$$

defines a Lorentz metric in $\mathscr{H}$.

We can interpret a solution $u$ of (1.2) as a scalar disturbance in a time varying anisotropic medium occupying $\boldsymbol{R}^{N}$. We study the energy of $u$ in a region of $\boldsymbol{x}$-space which expands at least as fast as wave-fronts for $L$. Let $S(T)$ be the region at time $T$; formally we consider $S(T)$ as a domain in the hyperplane $t=T$ in $\mathscr{H}$. As $T$ increases, the $S(T)$ sweep out a region $D(0, \infty)=\bigcup\{S(T): T>0\}$ in $\mathscr{H}$.

We say that region $S(T)$ expands faster than light, or faster than wavefronts for $L$, if the following two conditions are met: First, the boundaries $\partial S(T)$ sweep out a smooth hypersurface $S^{\prime}$ in $\mathscr{H}$, which is the lateral part of the boundary of $D(0, \infty)$. Second, the outer unit normal $\boldsymbol{n}=\left(n_{0}, n_{1}, \cdots, n_{N}\right)$ on $\partial D(0, \infty)$ satisfies $n_{0}<0$ and $((\boldsymbol{n}, \boldsymbol{n})) \geqq 0$ along $S^{\prime}$. For example, for a fixed $r$ the region $S(T)=\{(T, x):|x| \leqq M T+r\}$ expands faster than light. If $\boldsymbol{n}$ is negative characteristic on $S^{\prime}$, then the $\partial S(T)$ are an expanding wave-front for $L$.

Suppose that the region $S(T)$ expands at least as fast as light. If $w$ is a $C^{2}$ function in $\mathscr{H}$, we discuss its size in terms of the energy integral

$$
\mathscr{E}(w, T)=\int_{S(T)}\left(w^{2}+w_{t}^{2}+\sum_{i j=1}^{N} a_{i j} w_{, i} w_{, j}\right) d x .
$$

This paper gives conditions under which a solution $u$ of (1.2) will satisfy a lower bound of the form

$$
\mathscr{E}(u, T) \geqq C e^{-\gamma f(T)} \mathscr{E}(u, \tau)
$$


for $T>\tau \geqq 0$. In (1.4), $C$ and $\gamma$ are positive constants, and $f$ is a function which increases without bound as $T \rightarrow \infty$.

In particular we have the following results:

RESULT I. If $k_{1}(t, x)=O\left(t^{-2}\right), k_{2}(t, x)=O\left(t^{-1}\right)$, and all $\left|\left(a_{i j}\right)_{t}\right|=O\left(t^{-1}\right)$, then (1.4) holds with $f(T)=\ln (T)$.

RESULT II. If $k_{1}, k_{2}$, and all $\left|\left(a_{i j}\right)_{t}\right|$ are bounded, then (1.4) holds with $f(T)=T$.

RESULT III. If there is a constant $c>1$ such that $k_{1}(t, x)=O\left(t^{2 c-2}\right)$, $k_{2}(t, x)=O\left(t^{c-1}\right)$, and all $\left|\left(a_{i j}\right)_{t}\right|=O\left(t^{c-1}\right)$, then (1.4) holds with $f(T)=T^{c}$.

For derivatives we use the notation $\partial w / \partial t=w_{t}$ and $\partial w / \partial x_{i}=w_{, i}$. The gradient $\nabla w$ is taken with respect to all $N+1$ variables; and $|\nabla w|^{2}=$ $w_{t}^{2}+\sum_{i=1}^{N} w_{, i}^{2}$.

We introduce the quadratic form

$$
P_{b, c}(\xi)=2((b, \xi))((c, \xi))-((b, c))((\xi, \xi))
$$

for vector fields $\boldsymbol{b}, \boldsymbol{c}$, and $\boldsymbol{\xi}$ on $\boldsymbol{R} \times \boldsymbol{R}^{N}$. As shown by Hörmander [2], this form is positive definite if $\boldsymbol{b}$ and $\boldsymbol{c}$ are positive timelike vectors. [A vector $\boldsymbol{d}=\left(d_{0}, d_{1}, \cdots, d_{N}\right)$ is positive timelike iff $d_{0}>0$ and $((\boldsymbol{d}, \boldsymbol{d}))>0$.] Notice that $\boldsymbol{P}_{\boldsymbol{b}, \boldsymbol{c}}(\xi)$ is linear in $\boldsymbol{b}$ and $\boldsymbol{c}$. We use $\boldsymbol{h}$ to denote the timelike vector $\boldsymbol{h}=(1,0, \cdots, 0)$ in $\boldsymbol{R} \times \boldsymbol{R}^{N}$. Since $P_{h, h}(\xi)=\xi_{0}^{2}+\sum_{i, j=1}^{N} a_{i j} \xi_{i} \xi_{j},|\nabla w|$ and $\left(P_{h, h}(\nabla w)\right)^{1 / 2}$ are equivalent.

The starting point for the basic estimates is the formula

$$
\int_{D} 2 \lambda w_{t} L w=\int_{D}\left(P_{h, \nabla \lambda}(\nabla w)+\lambda \sum_{i j=1}^{N}\left(a_{i j}\right)_{t} w_{, i} w, j\right)-\int_{\partial D} \lambda P_{h, n}(\nabla w)
$$

which is valid for any $C^{1}$ function $\lambda=\lambda(t, x)$ and any $C^{2}$ function $w=$ $w(t, \boldsymbol{x})$ where $D$ is a bounded domain with piecewise smooth boundary and $n$ is the outer unit normal along $\partial D$. This formula follows directly from integration by parts.

2. Proof of Result II. In this section we consider (1.2) under the assumption that there are constants such that

$$
\left|\left(a_{i j}\right)_{t}\right| \leqq K ; \quad k_{1}(t, \boldsymbol{x}) \leqq K_{1} ; \quad k_{2}(t, \boldsymbol{x}) \leqq K_{2}
$$

in $\mathscr{H}$. We will prove Result II of the Introduction as Theorem 2.3. $\S 3$ will outline the slight modifications which adapt the proof to the hypotheses for Results I and II.

We start by developing the basic a priori inequality. Notice that no boundary conditions are imposed; the choice of domains and the properties of $P_{b, c}(\xi)$ combine to make boundary conditions unnecessary. 
Let $S(T)$ be a region in $x$-space expanding faster than light. Let $D(\tau, T)$ be the region in $\mathscr{H}$ swept out by the $S(t)$ for $\tau<t<T$; i.e.,

$$
D(\tau, T)=\{(t, x): x \in S(t) \text { and } \tau<t<T\} .
$$

Then the boundary $\partial D(\tau, T)$ is composed of three smooth pieces: $S(T)$, $S(\tau)$, and the lateral portion along $S^{\prime}$. The outer unit normal $n=$ $\left(n_{0}, n_{1}, \cdots, n_{N}\right)$ on $\partial D(\tau, T)$ is equal to $\boldsymbol{h}$ on $S(T)$ and to $-\boldsymbol{h}$ on $S(\tau)$.

Suppose $v$ is a $C^{2}$ function and $D$ is one of the regions $D(\tau, T)$ for $0 \leqq \tau<T$. We develop a weighted $L^{2}$ estimate for $v$ and $\nabla v$ in $D$ in terms of $L v$. To do this we introduce an auxiliary function $u^{*}=e^{\alpha t} v$ for $\alpha$ a positive parameter. Computation shows that

$$
e^{\alpha t} L v=L w+2 \alpha w_{t}-\alpha w^{2} .
$$

Using the elementary inequality $(X+Y+Z)^{2} \geqq 2 Y(X+Z)$ we get

$$
e^{2 \alpha t}|L v|^{2} \geqq 2\left(2 \alpha w_{t}\right)\left(L w-\alpha^{2} w\right) .
$$

For $\beta>0$, we multiply through by $e^{\beta t}$ and then integrate over $D$ to obtain

$$
\iint_{D} e^{\beta t} e^{2 \alpha t}|L v|^{2} \geqq 2 \alpha \iint_{D} 2 e^{\beta t} w_{t} L w-2 \alpha^{3} \iint_{D} e^{\beta t}\left(w^{2}\right)_{t} .
$$

Integration by parts and the properties of $n$ on $\partial D$ give us

$$
\begin{aligned}
-\iint_{I)} e^{\beta t}\left(w^{2}\right)_{t} & =\iint_{D} \beta e^{\beta t} w^{2}-\int_{\partial D} n_{0} e^{\beta t} w^{2} \\
& \geqq \beta \iint_{D} e^{\beta t} w^{2}+\int_{S(\tau)} e^{\beta t} w^{2}-\int_{S(T)} e^{\beta t} w^{2} .
\end{aligned}
$$

The next two lemmas provide an estimate for the other integral on the right side of (2.2).

Lemma 2.1. Suppose $\beta m^{2} \geqq 2 K N$. Then

$$
\begin{aligned}
& 2 \iint_{D} e^{\beta t} w_{t} L w \geqq \frac{1}{2} \beta \iint_{D} e^{\beta t} P_{h, h}(\nabla w)-\int_{S(T)} e^{\beta t} P_{h, h}(\nabla w) \\
& +\int_{S(\tau)} e^{\beta t} P_{h, h}(\nabla w) .
\end{aligned}
$$

Proof. If $\lambda=e^{\alpha t}$ and $D=D(\tau, T)$, then formula (1.5) specializes to

$$
2 \iint_{D} e^{\beta t} w_{t} L w+\int_{\partial D} e^{\beta t} P_{h, n}(\nabla w)=\iint_{D} e^{\beta t}\left\{\beta P_{h, h}(\nabla w)+\sum_{i j=1}^{N}\left(a_{i j}\right)_{t} w_{, i} w, j\right\} .
$$


By assumption (1.3) we have

But we also have

$$
P_{h, h}(\nabla w)=w_{t}^{2}+\sum_{i j=1}^{N} a_{i j} w_{, i} w_{, j} \geqq m^{2} \sum_{i=1}^{N}\left(w_{, i}\right)^{2} .
$$

$$
\begin{aligned}
\left|\sum\left(a_{i j}\right)_{t} w_{, i} w_{, j}\right| & \leqq \sum_{i j=1}^{N}\left|\left(a_{i j}\right)_{t}\right|\left|w_{, i}\right|\left|w_{, j}\right| \\
& \leqq K\left(\sum_{i=1}^{N}\left|w_{, i}\right|\right)^{2} \leqq K N \sum_{i=1}^{N}\left(w_{, i}\right)^{2}
\end{aligned}
$$

Since $\frac{1}{2} \beta m^{2} \geqq K N$, we can conclude that

$$
\beta P_{h, h}(\nabla w)+\sum\left(a_{i j}\right)_{t} w_{, i} w_{, j} \geqq \frac{1}{2} \beta P_{h, h}(\nabla w) .
$$

The properties of $\boldsymbol{n}$ on $\partial D$ yield the inequality

$$
\int_{\partial D} e^{\beta t} P_{h, n}(\nabla w) \leqq \int_{S(T)} e^{\beta t} P_{h, h}(\nabla w)-\int_{S(\tau)} e^{\beta t} P_{h, h}(\nabla w) .
$$

Using (2.6) and (2.7) to estimate terms in (2.5) we obtain the inequality of the lemma.

We now prove the basic a priori inequality.

THEOREM 2.2. Suppose $v$ is a $C^{2}$ function on $\bar{D}(0, \infty)$. If $\alpha>1, \beta m^{2} \geqq$ $2 K N$, and $0 \leqq \tau<T$, then there are constants $c_{i}$ such that

$$
\begin{aligned}
6 \alpha^{3} e^{(\beta+2 \alpha) T} \mathscr{E}(v, T) & +\iint_{D(\tau, T)} e^{(\beta+2 \alpha) t}|L v|^{2} \\
& \geqq c_{1} \alpha \cdot \beta \iint_{D(\tau, T)} e^{(\beta+2 \alpha) t}\left(v^{2}+|\nabla v|^{2}\right)+c_{2} e^{(\beta+2 \alpha) \tau} \mathscr{E}(v, \tau) .
\end{aligned}
$$

Proof. Let $D$ denote $D(\tau, T)$ and let $w=e^{\alpha t} v$. Then we can combine inequalities (2.3), (2.4), and (2.2) to obtain

$$
\begin{aligned}
& \iint_{D} e^{(\beta+2 \alpha) t}|L v|^{2}+2 \alpha \int_{S(T)} e^{\beta t}\left[P_{h, h}(\nabla w)+\alpha^{2} w^{2}\right] \\
& \quad \geqq \alpha \beta \iint_{D} e^{\beta t}\left[P_{h, h}(\nabla w)+2 \alpha^{2} w^{2}\right]+2 \alpha \int_{S(\tau)} e^{\beta t}\left[P_{h, h}(\nabla w)+\alpha^{2} w^{2}\right] .
\end{aligned}
$$

From the expression of $P_{h, h}(\nabla w)$ in terms of $v$ we can show that

$$
P_{h, h}(\nabla w) \leqq 2 e^{2 \alpha t}\left[\alpha^{2} v^{2}+P_{h, h}(\nabla v)\right]
$$


and

$$
P_{h, h}(\nabla w) \geqq e^{2 \alpha t}\left[-\frac{1}{2} \alpha^{2} v^{2}+\frac{1}{3} P_{h, h}(\nabla v)\right] .
$$

Thus (2.9) leads to the inequality

$$
\begin{aligned}
\iint_{D} e^{(\beta+2 \alpha) t}|L v|^{2}+ & 2 \alpha e^{(\beta+2 \alpha) T} \int_{S(T)}\left[3 \alpha^{2} v^{2}+P_{h, h}(\nabla v)\right] \\
\geqq & \alpha \beta \iint_{D} e^{(\beta+2 \alpha) t}\left[\frac{3}{2} \alpha^{2} v^{2}+\frac{1}{3} P_{h, h}(\nabla v)\right] \\
& +2 \alpha e^{(\beta+2 \alpha) \tau} \int_{S(\tau)}\left[\frac{1}{2} \alpha^{2} v^{2}+\frac{1}{3} P_{h, h}(\nabla v)\right] .
\end{aligned}
$$

The desired inequality (2.8) now follows because of the hypothesis $\alpha>1$, the equivalence of $P_{h, h}(\nabla v)$ with $|\nabla v|^{2}$, and the definition of $\mathscr{E}(v, T)$.

THEOREM 2.3. Suppose $u$ is a $C^{2}$ solution of (1.2) in the closure of $D(\tau, \infty)$. Then there are positive constants $C$ and $\gamma$, independent of $u$, such that

for all $T>\tau$.

$$
\mathscr{E}(u, T) \geqq C e^{-\gamma T} \mathscr{E}(u, \tau)
$$

Proof. Pick a fixed $\beta$ so that $\beta m^{2} \geqq 2 K N$. Since $u$ is a $C^{2}$ function we can apply Theorem 2.2. Let $D$ denote $D(\tau, T)$. For $\alpha>1$ and $T>\tau \geqq 0$, we have

$$
\begin{aligned}
6 \alpha^{3} e^{(\beta+2 \alpha) T} \mathscr{E}(u, T) & +\iint_{D} e^{(\beta+2 \alpha) t}|L u|^{2} \\
& \geqq c_{1} \alpha \beta \iint_{D} e^{(\beta+2 \alpha) t}\left(u^{2}+|\nabla u|^{2}\right)+c_{2} e^{(\beta+2 \alpha) \tau} \mathscr{E}(u, \tau) .
\end{aligned}
$$

From (1.2) and (2.1) we find

$$
\iint_{D} e^{(\beta+2 \alpha) t}|L u|^{2} \leqq 2 \iint_{D} e^{(\beta+2 \alpha) t}\left(K_{1}^{2} u^{2}+K_{2}^{2}|\nabla u|^{2}\right) .
$$

Let $K_{3}=\max \left\{K_{1}^{2}, K_{2}^{2}\right\}$. It follows that

$6 \alpha^{3} e^{(\beta+2 \alpha) T} \mathscr{E}(u, T) \geqq \iint_{D} e^{(\beta+2 \alpha) t}\left(c_{1} \alpha \beta-2 K_{3}\right)\left(u^{2}+|\nabla u|^{2}\right)+c_{2} e^{(\beta+2 \alpha) r} \mathscr{E}(u, \tau)$.

Setting $\gamma=\beta+2 \alpha$ for $\alpha$ sufficiently large we have

$$
6 \alpha^{3} e^{\gamma T} \mathscr{E}(u, T) \geqq c_{2} e^{\gamma \tau} \mathscr{E}(u, \tau) .
$$

The choice of $C$ required for the theorem is now apparent. 
COROLLARY. If $u$ is a nonnull solution of $(1.2)$ in $\bar{D}(\tau, \infty)$, then $\mathscr{E}(u, T)$ cannot decay faster then $e^{-\gamma T}$, where $\gamma$ is chosen as above.

Proof. Let $C$ and $\gamma$ be taken as in the theorem. If $\tau<\sigma<T$, then the proof of the theorem also shows that

$$
\mathscr{E}(u, T) \geqq C e^{-\gamma T} \mathscr{E}(u, \sigma) .
$$

So if $\lim _{T \rightarrow \infty} e^{\gamma T} \mathscr{E}(u, T)=0$, then $\mathscr{E}(u, \sigma)=0$ for all $\sigma>\tau$. This would show that $u \equiv 0$ in $\bar{D}(\tau, \infty)$.

3. Remarks on Results I and III. This section indicates how the proof of Theorem 2.3 is adapted to prove Results I and III. Again we consider a given family of regions $S(T)$ expanding faster than light.

THEOREM 3.1. Suppose the coefficients of (1.2) satisfy the bounds

$$
\left|\left(a_{i j}\right)_{t}\right| \leqq K t^{-1} ; \quad k_{1}(t, x) \leqq K_{1} t^{-2} ; \quad k_{2}(t, x) \leqq K_{2} t^{-1}
$$

in $\bar{D}(\tau, \infty)$ for some $\tau>0$. Suppose $u$ is a solution of $(1.2)$ in $\bar{D}(\tau, \infty)$. Then there are constants $C$ and $\gamma$, independent of $u$, such that

$$
\mathscr{E}(u, T) \geqq C T^{-\gamma} \mathscr{E}(u, \tau) \text { for all } T>\tau .
$$

Proof. The pattern of proof follows that of $\$ 2$ exactly except that the weight functions $\lambda=e^{\alpha t}$ are replaced by $\lambda=\exp (\alpha \ln (t))=t^{\alpha}$. The decay conditions imposed on the coefficients of (1.2) by (3.1) are those required so that the weighted $L^{2}$ integral for $L u$ on $D(\tau, T)$ can be dominated by the terms in $u^{2}$ and $P_{h, h}(\nabla u)$ which arise from the a priori estimate analogous to Theorem 2.2.

THEOREM 3.2. Suppose there is a constant $c>1$ such that the coefficients of (1.2) satisfy the bounds

$$
\left|\left(a_{i j}\right)_{t}\right| \leqq K t^{c-1} ; \quad k_{1}(t, x) \leqq K_{1} t^{2 c-2} ; \quad k_{2}(t, x) \leqq K_{2} t^{c-1}
$$

in some $\bar{D}(\tau, \infty)$. Suppose that u satisfies $(1.2)$ in $\bar{D}(\tau, \infty)$. There are constants $C$ and $\gamma$, independent of $u$, such that

$$
\mathscr{E}(u, T) \geqq C \exp \left(-\gamma T^{c}\right) \mathscr{E}(u, \tau) \text { for all } T>\tau .
$$

Proof. Here again we follow the outline of $\$ 2$ using this time the weight functions $\lambda=\exp \left(\alpha t^{c}\right)$ in place of $\lambda=e^{\alpha t}$.

\section{REFERENCES}

1. C. O. Bloom and N. D. Kazarinoff, Energy decay exterior to star-shaped obstacles in energy-active media, Notices Amer. Math. Soc. 19 (1972), A-141. Abstract \#691-35-16.

2. L. Hörmander, Uniqueness theorems for normally hyperbolic partial differential equations of the second order, Tolfte Skand. Math. Kongr., Lund, 1953, pp. 105-115. MR 16, 483. 
3. H. A. Levine, Some uniqueness criteria in the Cauchy problem for $P u_{t t}+M u_{t}+$ $N u=0$ in Hilbert space, Battelle (Geneva) Advanced Studies Center Report 52, 1971.

4. A. C. Murray, Asymptotic behavior of solutions of hyperbolic inequalities, Trans. Amer. Math. Soc. 157 (1971), 279-296. MR 43 \#680.

5. H. Ogawa, Lower bounds for solutions of hyperbolic inequalities, Proc. Amer. Math. Soc. 16 (1965), 853-857. MR 33 \#1596.

6. M. H. Protter, Asymptotic behavior and uniqueness theorems for hyperbolic operators, Outlines Joint Sympos. Partial Differential Equations (Novosibirsk, 1963), Acad. Sci. USSR Siberian Branch, Moscow, 1963, pp. 348-353. MR 34 \#1659.

Department of Mathematics, Cornell University, Ithaca, New York 14850

Current address: Department of Mathematics, Douglass College, Rutgers University, New Brunswick, New Jersey 08903 